\title{
anatomy
}

Teaching Anatomy

www.anatomy.org.tr

Received: Fanuary 8, 2019; Accepted: February 15, 2019

doi:10.2399/ana.19.016

\section{Impact of a gynecologic oncology cadaveric dissection course for surgical training}

\author{
İlker Selçuk ${ }^{1}$ (D), Çağatay Barut ${ }^{2}$ (D), Eray Çalışkan ${ }^{3}$ (D) \\ ${ }^{1}$ Department of Gynecologic Oncology, University of Health Sciences, Zekai Tabir Burak Woman's Health Education and Research Hospital, Ankara, Turkey \\ ${ }^{2}$ Department of Anatomy, Bahçesebir University Faculty of Medicine, Istanbul, Turkey \\ ${ }^{3}$ Department of Obstetrics and Gynecology, Okan University Faculty of Medicine, Istanbul, Turkey
}

\begin{abstract}
Objectives: The aim of this study was to measure the educational efficacy of a gynecologic oncology cadaveric dissection course on fellows and specialists.

Methods: After the radical and reconstructive vulvar and abdominal gynecologic cancer surgery cadaveric course, a post-course survey was applied to evaluate the improvement in topographic surgical anatomy and the effect on broadening the surgical experience.

Results: Totally 10 and 16 participants attended to the vulvar cancer surgery cadaveric dissection course and abdominal gynecologic cancer surgery cadaveric dissection course, respectively. All participants stated that they had an improvement in topographic surgical anatomy and they found the cadaveric workshop beneficial to broaden the surgical experience. All participants suggested this kind of courses to learn the proper techniques of a surgical procedure in a comfortable and non-stressful setting and improve the surgical skills for rare and complicated surgeries.
\end{abstract}

Conclusion: Cadaveric dissection courses in the field of gynecologic oncology tailor the surgical anatomy education and improve the training.

Keywords: anatomy; cadaveric dissection; education; gynecologic oncology; surgery

Anatomy 2019;13(1):56-60 @2019 Turkish Society of Anatomy and Clinical Anatomy (TSACA)

\section{Introduction}

There is a trend shifting towards problem-based learning in surgical anatomy education. Facing with difficult cases increased the need for a detailed knowledge of anatomy, and many residents and junior post-graduate surgeons know little about the relevant anatomical planes of the procedure they perform. ${ }^{[1]}$ Many post-graduate surgical anatomy courses which are organized nowadays brought the solution to this problem.

Cadaveric dissection constitutes the cornerstone of anatomy education since centuries; however, integration of $3 \mathrm{D}$ printed models and computer-based virtual and augmented reality programs improved the general teaching and learning utilities which lead to a more structured design of education. ${ }^{[2,3]}$ Despite the improvements in software programs and models, cadaveric dissection provides a different point of view especially for surgical education and training. ${ }^{[4]}$

Researchers in the field of anatomy investigated the role of many teaching tools like software, advanced imaging or multimedia-based programs, 3D printed material; however, it is widely accepted that there is no one particular way of teaching anatomy. ${ }^{[5]}$ Combination of traditional and novel innovative methods provides an excellent way of teaching and learning anatomy; nonetheless, the group you intend to teach is the major determinant for the way you need to select during anatomy education. ${ }^{[6]}$ In that point, the educational activities differ due to the target group. Surgeons need to learn the 3D oriented anatomy and close anatomical landmarks of the field they operate, and management of potential complications, additionally they need to practice to gain skills and confidence. On the basis of these needs, the cadaveric dissection studies give them 
many vulnerable options. This study evaluated the educational results of a 'Radical and reconstructive vulvar and abdominal surgery cadaveric course' on learning anatomy and gaining surgical experience.

\section{Materials and Methods}

Second annual cadaveric course on radical surgical procedures in gynecologic oncology was held in September 2018 at Department of Anatomy, Faculty of Medicine in Bahçeşehir University. The venue was in Istanbul, an ancient city with lots of historical and modern sightseeing places.

The course was separated into two workshops; Radical and Reconstructive Vulvar Cancer Surgery Workshop on August 31, 2018 and Radical and Reconstructive Abdominal Gynecologic Cancer Surgery Workshop on September 1-2, 2018. Both were European Society of Gynecological Oncology (ESGO) endorsed meetings. Professor Ate Van der Zee, Professor Christina Fotopoulou (ESGO Council Member) and Dr. Kamil Zalewski (President of European Network of Young Gynae-Oncologists) were the international faculty members, with collaboration of 20 national (Turkish) faculty members. Turkish Society of Obstetrics and Gynecology supervised the courses. Department of Anatomy in Bahçeşehir University supported the workshop and a clinical anatomist - Professor Çağatay Barut - was the faculty member both for theoretical lessons and cadaveric dissection. Ten participants attended to the vulvar cancer workshop in which three totally fresh-frozen cadavers were used; 16 participants attended to the abdominal surgery workshop in which four totally fresh-frozen cadavers were used. A heterogeneous group of gynecologic oncology fellows and specialists from Turkey, Azerbaijan and Europe attended the course.

The vulvar cancer workshop was a one-day program. Theoretical lessons finished in early afternoon and three hours were spent in the anatomy laboratory for cadaveric dissection. Radical vulvectomy and inguinofemoral lymph node dissection with alternative flap reconstructions were performed at the master table; so, all the participants watched the operation live for this rare vulvar cancer surgery by a video recording system. Afterwards, participants performed the dissection and surgical procedures under the supervision of seniors (Figure 1).

Radical abdominal surgery workshop consisted of theoretical lessons for the first day and cadaveric dissection for the whole second day. Theoretical lessons covered all the advanced procedures for pelvis and upper abdomen with special sessions targeting ovarian cancer surgery, management of complications and reconstruction techniques. At the end, all the faculty discussed 'what is not written in books, tips and tricks of surgical principles' interactively. Coffee breaks were beneficial for networking and a specially organized dinner was held after the end of first and second days which presented the beauty and history of Bosphorus in a lovely climate.

Second day focused on practical sessions with four fresh frozen cadavers covering all the open surgical procedures that will be applied in open abdomen. Additionally, participants had enough time to make dissections and perform the surgical procedures for tailoring the surgical practice. Anatomy laboratory was well designed to maintain a clean working environment. Table 1 shows the subjects of cadaveric dissections.

After the end of the course, a questionnaire was replied by all of the participants to measure the efficacy of the course, with the answers 'Yes' or 'No'. The post-

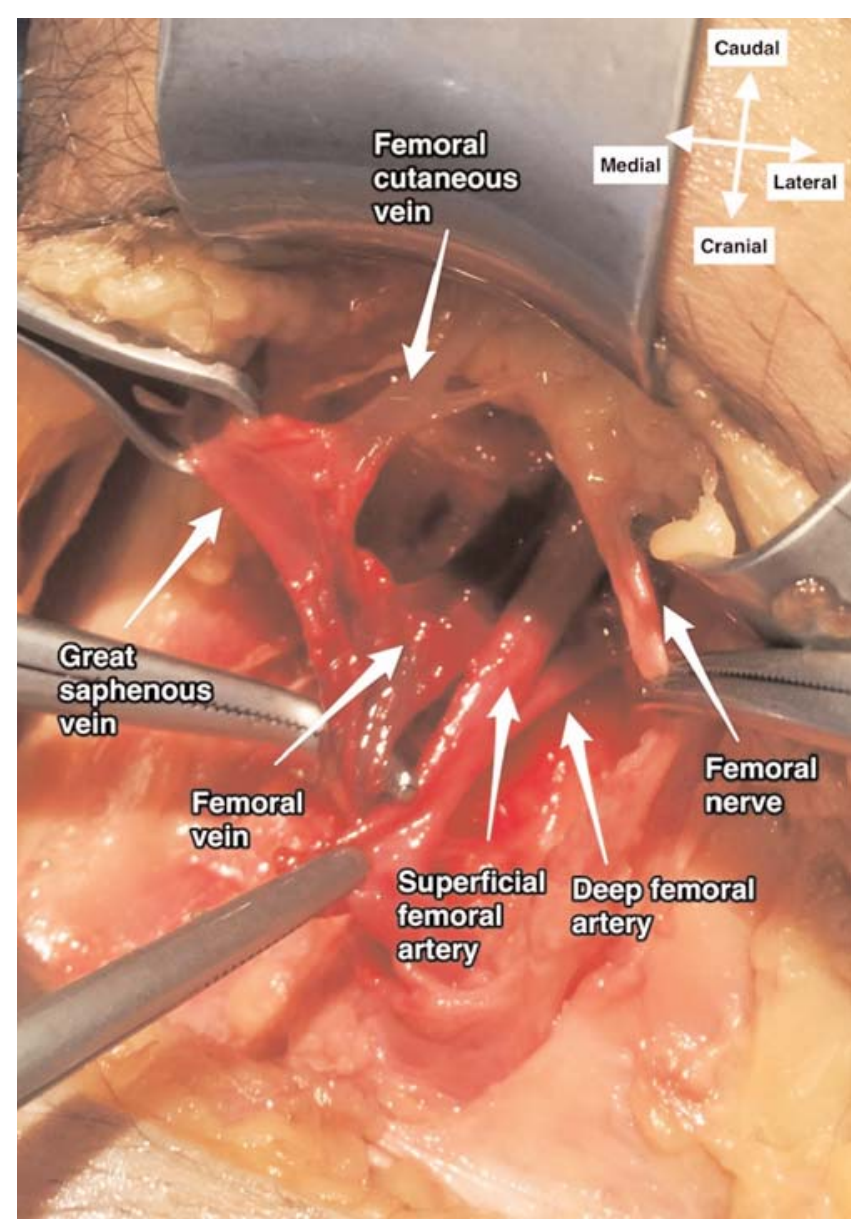

Figure 1. Right inguinofemoral lymph node dissection and femoral vessels on a fresh-frozen cadaver. [Color figure can be viewed in the online issue, which is available at www.anatomy.org.tr] 
Table 1

Subjects of procedures performed during cadaveric dissections.

Vulvar cancer surgery cadaveric workshop

- Vulvar dissection

- Radical vulvectomy and wide local excision

- Skinning vulvectomy

- Dissection of uretra and anus, excision and repair

- Inguino-femoral dissection, femoral vessels

- Vaginal surgery

- Vulvar/vaginal reconstruction

- Vulvar flaps and step by step flap techniques

Abdominal gynecologic cancer surgery cadaveric workshop (open surgical procedures)

- Anatomy of abdominal wall, different incision types

- Mobilizations of right and left hepatic lobe

- Identification and dissection of hepatoduodenal ligament and portal triad

- Pringle maneuver

- Step by step segmental hepatectomy and pont hepatique

- Cholecystectomy

- Diaphragm stripping and resection

- Repair of diaphragm and chest tube insertion

- Cardiophrenic lymph node excision

- Mobilization of stomach, dissection of subpyloric area and partial gastric resection

- Bursa omentalis, lesser sac and celiac trunk dissection

- Splenectomy and pancreas tail excision

- Omentectomy

- Retroperitoneal right side medial visceral rotation; Kocher and Cattel-Braasch maneuver

- Retroperitoneal left side medial visceral rotation, Mattox maneuver

- Resection/anastomosis of small and large bowel, rectum

- Techniques in opening stoma

- Mobilization of kidney, nephrectomy and adrenal gland resection

- Pelvic lymphadenectomy

- Para-aortic lymphadenectomy

- Radical hysterectomy and nerve sparing radical hysterectomy

- Total mesometrial resection

- Laterally extended endopelvic resection

- Trachelectomy

- Radical oophorectomy en-bloc rectosigmoid resection and pelvic peritonectomy

- Total pelvic exenteration (isolated anterior/posterior)

- Mobilization and injury repair of vena cava, aorta and common iliac vessels

- Partial resection and repair of ureter and bladder

- Radical cystectomy

- Continent urinary pouch and neo-bladder course survey aimed to measure the improvement in topographic surgical anatomy and surgical experience. Results were analyzed with a computer-based calculation system.

\section{Results}

All of the participants, 10 for the Radical and Reconstructive Vulvar Cancer Surgery Workshop and 16 for the Radical and Reconstructive Abdominal Gynecologic Cancer Surgery Workshop, stated that they had an improvement in topographic surgical anatomy $(100 \%)$ and found the cadaveric workshop beneficial to broaden the surgical experience (100\%) (Table 2).

Additionally, all of the participants suggested this kind of courses to learn the proper techniques of a surgical procedure in a comfortable and non-stressful setting (100\%) and improve the surgical skills for rare and complicated surgeries (100\%) (Table 2).

\section{Discussion}

Irrespective of the medical specialty, anatomy education is the supplementary part of clinical practice. Despite many ways of teaching anatomy, simulation based hands-on models are becoming an increasing demand among the surgical residents and post-graduate surgeons. There are many deficiencies that surgical residents come across during the residency or post-residency. Most importantly, feeling fully prepared to perform a surgical procedure or manage the surgery independently is lacking among the junior surgeons. This deficiency arises from the gap in anatomy education and projecting the anatomic knowledge into surgical practice. ${ }^{[7-9]}$ This study focuses on the effect of gynecologic oncology cadaveric dissection course on surgical anatomy knowledge and practice.

The results of this study revealed that a single cadaveric course improves the topographic surgical anatomy knowledge and broaden the surgical experience. However,

Table 2

Post-course evaluation of educational efficacy

\begin{tabular}{lcccc}
\hline & \multicolumn{2}{c}{ Vulvar cancer workshop $(\mathbf{n}, \%)$} & & Abdominal surgery workshop (n, \%) \\
\cline { 2 - 3 } & Yes & No & Yes \\
\hline Improvement in topographic surgical anatomy & $10(100 \%)$ & None & $16(100 \%)$ & None \\
\hline Beneficial to broaden the surgical experience & $10(100 \%)$ & None & $100 \%)$ & None \\
\hline $\begin{array}{l}\text { Suggest this course to learn proper techniques of surgical } \\
\text { procedures in a comfortable and non-stressful setting }\end{array}$ & $10(100 \%)$ & None & $16(100 \%)$ & None \\
\hline $\begin{array}{l}\text { Suggest this course to improve surgical skills for rare and } \\
\text { complicated surgeries }\end{array}$ & $10(100 \%)$ & None \\
\hline
\end{tabular}


the limited number of the study population and measuring the effectivity subjectively are the major limitations of this study.

The practical way of surgical training is shaped on a learning method in which the skills are gathered from the senior surgeons during the operations performed on patients. The educational curriculum of surgical residency does not involve anatomy rotation and the ethical dilemma of performing a procedure for the first time on a patient reveals restrictions in surgical education. First of all, residents watch the surgical operations many times, afterwards they assist the first surgeon, lately they perform the procedure under mentorship and finally they perform the procedure independently. However, occasionally they do not find the opportunity to perform a surgical procedure independently. The study by Gultekin et al. ${ }^{[10]}$ which analyzed the gynecologic oncology training systems in Europe showed that many of the countries did not have a dedicated gynecologic oncology training program (47\%) and $41 \%$ of the fellows do not have an access to advanced laparoscopic surgical procedures. This brings the feeling of anxiety during the junior post-graduate period while operating. Moreover, many of the residents do not observe management of complications during the surgery, by the way they will be unexperienced to manage a difficult case or complication during the surgery.

Post-graduate courses are held with the aim of improving the surgical anatomy knowledge and surgical skills. Nevertheless, the most beneficial courses which contribute to the surgical education are hands-on practical activities. Cadaveric dissection courses are the practical way of understanding the anatomy and surgical planes that the participants watch the anatomical dissection of the mentor and they find the chance to self-practice and explore. On the other hand, the difficulties to maintain a cadaver, high-costs and limited number of participants per cadaver are the major drawbacks of cadaveric dissection courses.

Barton et al. ${ }^{[1]}$ stated that there was a significant improvement in surgical anatomy knowledge of pelvis and upper abdomen among the gynecologic oncology fellows after the cadaveric workshop of surgical procedures in gynecologic oncology and all of the participants found that the course was valuable and recommendable. After the anatomy of complications workshop to improve the training of gynecologic oncology fellows, Hammond et al. ${ }^{[12]}$ reported that cadaveric dissection courses provided practice of less common surgical procedures and assisted to learn how to manage complications. Additionally, Hammond et al. ${ }^{[13]}$ performed a similar study to shape an educational program for obstetricians and gynecologists. They designed a course based on interactive surgical anatomy lectures, practical surgery videos, cadaveric dissection and specific learning tasks, and aimed to teach how to deal with unexpected injuries and complications via cadaveric dissection. This combination of observational and practical learning improved the knowledge, confidence, competence and performance. Heisler ${ }^{[14]}$ emphasized the point of gross anatomy education at the laboratory which maintains discovering the anatomic landmarks and their relationships, developing tissue planes, good handling of instruments and self-dissection under mentorship. Despite the lacking objective criteria to evaluate the clinical application of gross anatomy education, there are many evidences that a structured anatomy education during junior residency or post-graduate period will improve the anatomical competence and surgical capability. ${ }^{[15,16]}$

There may be an option to mimic some surgical procedures where a fully-equipped material defining nearby anatomical landmarks is not needed, like performing a loop electrosurgical excision procedure. ${ }^{[17]}$ On the other hand, gynecologic oncology practice is related with performing major surgical procedures on abdominal visceral organs and aorta or inferior vena cava or branches and tributaries of them. Based on this reality, simulating operations like retroperitoneal lymphadenectomy, splenectomy, bowel or liver mobilization, or resection after developing the accurate surgical planes is not easily applicable on models or will not be highly-effective, because these procedures need the three-dimensional view of relevant anatomic structures and dissection of surgical planes. Procedure-based learning provides many gains for the residents and fellows, therefore cadaveric dissections will yield an important area of self-practice for particular surgical procedures. ${ }^{[18]}$ From a different point of view, simulation models or animal models will generate an area of practice for some abdominal procedures like ureteric or vascular repair or bowel anastomosis. ${ }^{[12]}$ Thus, the target population, aim of the course and selected procedures are the key elements to determine the style of teaching. This study was based on a cadaveric model to teach the vulvar and abdominal surgical procedures after dissection of all relevant surgical spaces and planes.

Cadaveric dissection courses may also tailor the surgical practice due to the needs of participants and may focus on a specific issue by performing proper dissections. Cadaveric dissection improves the basic surgical skills and confidence; yet, transfer of skills from the laboratory to the operation room is questionable. It is not easy to evaluate the gains which were reflected to the operation room while performing a procedure independently. Considering 
this, the degree and level of the skills and abilities transferred to the operation room are subjective and not totally measurable. ${ }^{[19,20]}$ However, it is obvious that cadaveric dissection courses improve the topographic surgical anatomy knowledge and dissection techniques used in surgery to develop proper tissue planes, which is the main result of this study.

One important issue as the limitation of cadaveric courses is that, since a single cadaver is shared by three or four participants, generally none of the participants complete all the steps of a single surgical procedure individually. However, all of the participants observe the master table cadaveric dissection, perform dissections under mentorship and apply the procedures by sharing the steps.

\section{Conclusion}

Cadaveric dissections improve the anatomical knowledge and surgical experience. However, there is a need for further investigations to demonstrate the objective efficacy of these courses.

\section{References}

1. Waterston SW, Stewart IJ. Survey of clinicians' attitudes to the anatomical teaching and knowledge of medical students. Clin Anat 2005;18:380-4.

2. Ghosh SK. Cadaveric dissection as an educational tool for anatomical sciences in the 21st century. Anat Sci Educ 2017;10:286-99.

3. Turney BW. Anatomy in a modern medical curriculum. Ann R Coll Surg Engl 2007;89:104-7.

4. Selcuk I, Tatar I, Huri E. Cadaveric anatomy and dissection in surgical training. Turk J Obstet Gynecol 2019;16:72-5.

5. Hu M, Wattchow D, de Fontgalland D. From ancient to avantgarde: a review of traditional and modern multimodal approaches to surgical anatomy education. ANZ J Surg 2018;88:146-51.

6. Sbayeh A, Qaedi Choo MA, Quane KA, Finucane P, McGrath D, O'Flynn S, O'Mahony SM, O'Tuathaigh CM. Relevance of anatomy to medical education and clinical practice: perspectives of medical students, clinicians, and educators. Perspect Med Educ 2016;5: $338-46$.

7. Fitzgerald JE, White MJ, Tang SW, Maxwell-Armstrong CA, James DK. Are we teaching sufficient anatomy at medical school? The opinions of newly qualified doctors. Clin Anat 2008;21:718-24.
8. Wynn GR, Austin RCT, Motson RW. Using cadaveric simulation to introduce the concept and skills required to start performing transanal total mesorectal excision. Colorectal Dis 2018;20:496-501.

9. Takayesu JK, Peak D, Stearns D. Cadaver-based training is superior to simulation training for cricothyrotomy and tube thoracostomy. Intern Emerg Med 2017;12:99-102.

10. Gultekin M, Dursun P, Vranes B, Laky R, Bossart M, Grabowski JP, et al. Gynecologic oncology training systems in Europe: a report from the European network of young gynaecological oncologists. Int J Gynecol Cancer 2011;21:1500-6.

11. Barton DP, Davies DC, Mahadevan V, Dennis L, Adib T, Mudan S, Sohaib A, Ellis H. Dissection of soft-preserved cadavers in the training of gynaecological oncologists: report of the first UK workshop. Gynecol Oncol 2009;113:352-6.

12. Hammond I, Taylor J, Obermair A, McMenamin P. The anatomy of complications workshop: an educational strategy to improve the training and performance of fellows in gynecologic oncology. Gynecol Oncol 2004;94:769-73.

13. Hammond I, Taylor J, McMenamin P. Anatomy of Complications workshop: an educational strategy to improve performance in obstetricians and gynaecologists. Aust N Z J Obstet Gynaecol 2003;43:111-4.

14. Heisler CA. Importance of adequate gross anatomy education: the impact of a structured pelvic anatomy course during gynecology fellowship. Anat Sci Educ 2011;4:302-4.

15. Kenton K, Mueller ER, Graziano S, Summers S, Rickey L, Oldham L, Pombar X, Turner F, Darrell B. Clinical anatomy and surgical skills training (CASST): development of a multicenter, multidisciplinary program. Am J Obstet Gynecol 2006;195:1789-93.

16. Corton MM, Wai CY, Vakili B, Boreham MK, Schaffer JI, Coleman RL. A comprehensive pelvic dissection course improves obstetrics and gynecology resident proficiency in surgical anatomy. Am J Obstet Gynecol 2003;189:647-51.

17. Connor RS, Dizon AM, Kimball KJ. Loop electrosurgical excision procedure: an effective, inexpensive, and durable teaching model. Am J Obstet Gynecol 2014;211:706 e1-3.

18. Hamstra SJ, Dubrowski A, Backstein D. Teaching technical skills to surgical residents: a survey of empirical research. Clin Orthop Relat Res 2006;(449):108-15.

19. Gilbody J, Prasthofer AW, Ho K, Costa ML. The use and effectiveness of cadaveric workshops in higher surgical training: a systematic review. Ann R Coll Surg Engl 2011;93:347-52.

20. Robinson DA, Piekut DT, Hasman L, Knight PA. Cadaveric Simulation training in cardiothoracic surgery: a systematic review. Anat Sci Educ 2019 Jun 24. doi: 10.1002/ase.1908.

Correspondence to: Ilker Selçuk, MD

Department of Gynecologic Oncology, University of Health Sciences, Zekai Tahir Burak Woman's Health Education and Research Hospital, Ankara, Turkey Phone: +90 5302010546 e-mail: ilkerselcukmd@hotmail.com

Conflict of interest statement: No conflicts declared.

This is an open access article distributed under the terms of the Creative Commons Attribution-NonCommercial-NoDerivs 3.0 Unported (CC BY-NCND3.0) Licence (http://creativecommons.org/licenses/by-nc-nd/3.0/) which permits unrestricted noncommercial use, distribution, and reproduction in any medium, provided the original work is properly cited. Please cite this article as: Selçuk İ, Barut Ç, Çalışkan E. Impact of a gynecologic oncology cadaveric dissection course for surgical training. Anatomy 2019;13(1):56-60. 